\title{
Molecular Junctions: From Tunneling to Function
}

\author{
Michel Calame*
}

\begin{abstract}
Thanks to the development of appropriate experimental techniques, molecular devices and their electrical transport properties have recently been the focus of a major research effort. This brief review describes how individual molecules can be contacted with metallic electrodes to form molecular junctions and addresses their basic formation mechanisms. An extension to molecular junctions networks is also discussed. Functionality could be demonstrated in such systems, and examples where conductance modulation using light or chemical stimuli was achieved will be presented.
\end{abstract}

Keywords: Break junctions · Molecular junction · Molecular switch · Nanoelectronics · Nanoparticles arrays

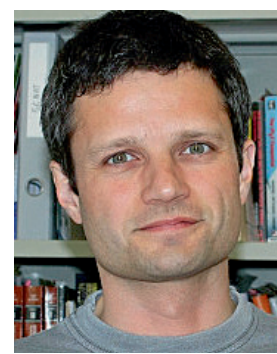

After graduating in physics from the University of Neuchâtel in Switzerland, $\mathbf{M i}$ chel Calame started a $\mathrm{PhD}$ in condensed matter physics under the guidance of Prof. P. Martinoli in 1993. His PhD work involved the fabrication of thin superconducting films and the study of their electrical transport properties in presence of magnetic field at cryogenic temperatures. The interplay between the film thickness and the resulting vortex dynamics was the main focus of this research work. In 1998, Michel Calame spent a few months at the Swiss Federal Office of Metrology METAS (Bern, Switzerland) as a scientific collaborator, working on low-temperature electronics for single-electron transistor devices, before moving to the Center for Studies in Physics and Biology at the
Rockefeller University (NY, USA). There, he joined the group of Prof. A. Libchaber for a postdoctoral stay in molecular biophysics, studying the properties of fluorescent DNA oligomers (molecular beacons). At the end of 2000, he joined the nanoelectronics group of Prof. C. Schönenberger at the University of Basel. Since 2001, he has been a senior scientist and lecturer at the University of Basel, exploring the electrical properties of nanometer-scale structures with a particular emphasis on molecular devices. In 2007, he was elected at the Swiss Academy of Sciences, as a board member of the Mathematics, Astronomy and Physics Platform.

\section{Introduction}

In its broad acceptance, the term molecular electronics refers to the electronic functionality of systems involving organic crystals or polymers assemblies as the ac- tive part. Organic light-emitting diodes are one example among the devices emerging from this technology. ${ }^{[1]}$ Molecular electronics nowadays rather refers to electronic systems aimed at exploiting the properties of a small number, or even a single, molecule(s) comprising a few tens of atoms only. [2] The elementary unit in such devices can be termed a molecular junction (Fig. 1a) and this expression refers not only to the molecules forming the junction but also to the atomic-sized electrodes enabling their contact.[3] To some level, molecules, as well as metallic and semiconducting clusters, can be seen as the smallest building blocks where structure and electronic or electro-optical properties can be engineered. ${ }^{[4]}$ These nanoscale blocks can be chemically synthesized with atomic precision in large quantities and possess self-assembling properties. Building molecular electronic systems is therefore an interdisciplinary endeavor, consisting in combining conventional micro- and

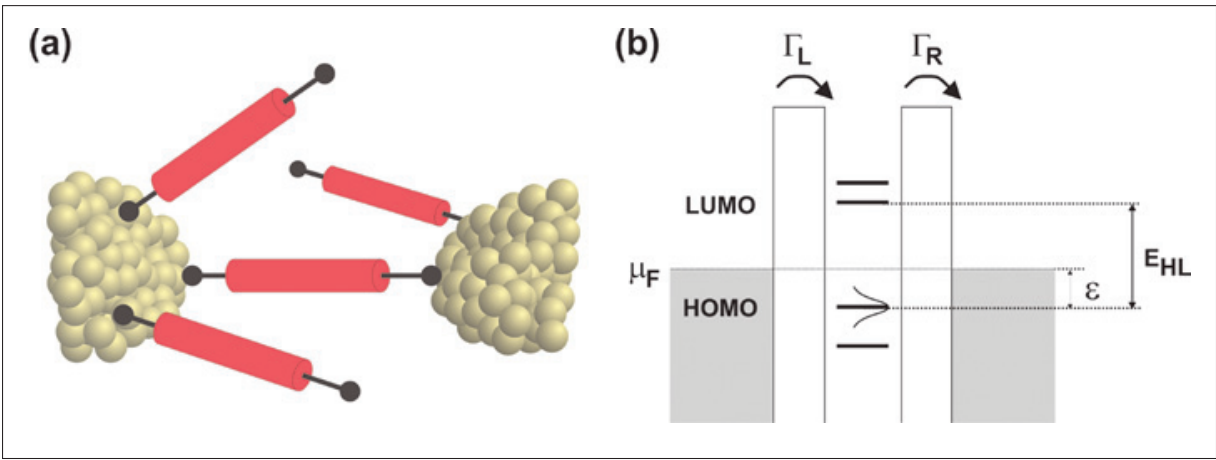

Fig. 1. (a) Representation of contact electrodes and individual molecules (red) forming a molecular junction. At the atomic scale, the electrodes will present some roughness while the molecules in the gap can adopt different orientations and binding conformations, depending on the anchor group and the electrode material. (b) Simplified energy diagram of a molecular junction at zero bias voltage. The thermal broadening of the molecular orbitals is schematically depicted for the HOMO level. $\Gamma_{L}$ and $\Gamma_{\mathrm{R}}$ are the left and right contacts coupling constants respectively and $\mu_{\mathrm{F}}$ is the electrochemical potential of the electrodes. 
nanofabrication techniques with chemical bonding and self-assembly processes.

Electron transfer processes are ubiquitous in nature and take place in a variety of physical, chemical and biological processes. Charge transport phenomena in semiconductor physics, corrosion reactions in chemistry or photosynthesis in biological systems are examples where electron transfer plays a central role. It was however probably only in the 1960s that the idea of combining solid-state electrodes with organic compounds emerged. Investigating semiconducting organic species and, later, nucleic acids, Eley and Spivey wondered what the transport through a single nucleic acid filament would be, provided one might be able to contact it. ${ }^{[5]}$ They anticipated that the overlap of $\pi$-orbitals stemming from the stacked structure of the oligonucleotides might lead to the formation of a conduction channel. Interestingly, the stacking of conjugated organic compounds embedded in molecular junctions has been demonstrated experimentally only recently, as will be illustrated below. In one of the first realizations of a hybrid device, combining solid-state electrodes and organic species, Mann and Kuhn studied junctions comprising monolayers of carboxylic chains sandwiched between two metal electrodes. ${ }^{[6]}$ They observed that the conductance of the junction decayed exponentially with the length of the monolayer thickness and could be described by quantum-mechanical tunneling. From a molecular electronics perspective, changing the monolayer thickness was an efficient way to tune the properties of their device: they successfully created a controllable molecular tunnel junction. It was however in 1974 that Aviram and Ratner anchored the idea that implementing design at the molecular level could lead to rectification as well as more advanced electronic functions in devices operating at the single molecule level.[7] The equilibrium energy diagram of a molecular junction can be represented schematically as in Fig. 1b. In a first approximation, the system can be seen as a set of molecular orbitals connected to a left and a right electron reservoir via the coupling constants $\Gamma_{\mathrm{L}}$ and $\Gamma_{\mathrm{R}}$. Besides the coupling strength, parameters such as the charging energy, the temperature or the relative position $\varepsilon$ of the molecular orbital relative to the electrochemical potential $\mathrm{E}_{\mathrm{F}}$ of the electrodes will define the transport regime of the junction, see $e . g$. refs [8-12]. Achieving an electrical functionality therefore consists in observing a conductance modulation effect related for instance to a change in the molecular orbitals arrangement in the junction. For this purpose, it is necessary to implement an additional control using for instance a

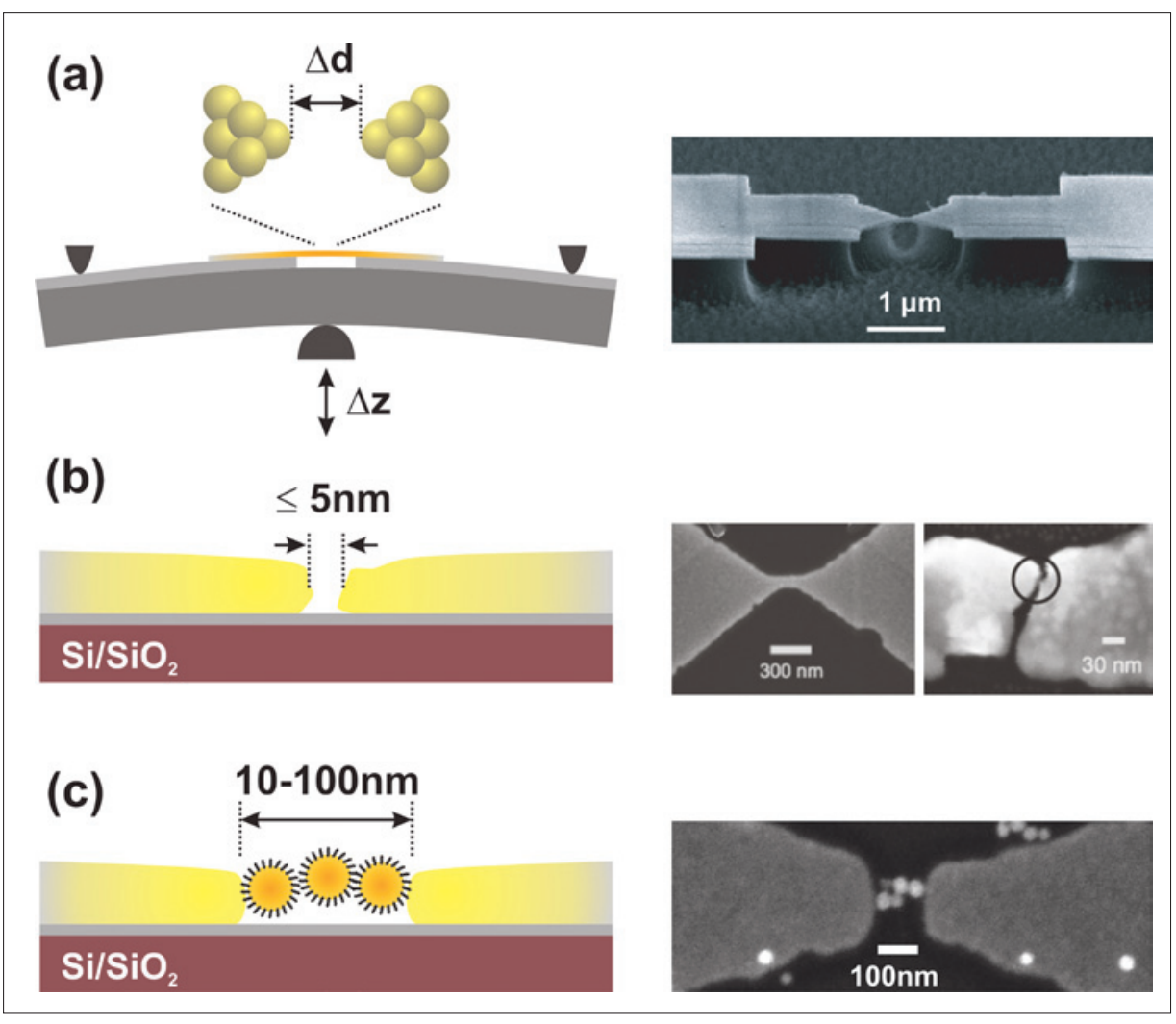

Fig. 2. Examples of strategies enabling the preparation of controlled nanoscale gaps between metallic electrodes. (a) Mechanically controllable break junction: a free-standing metallic bridge prepared on a flexible stainless-steel substrate (SEM image) is mounted in a three-point bending mechanism. The bridge can be elongated until it breaks open to form atomic-size contacts. (b) By inducing the migration of metallic atoms using a large current density (electromigration), a gap can be opened in metallic constrictions directly fabricated on a $\mathrm{Si} / \mathrm{SiO}_{2}$ substrate (SEM images: left, Au constriction; right, electromigration-induced slit and gap. The gap location is circled). (c) Dielectrophoretically trapped Au nanoparticles $(\varnothing \sim 30 \mathrm{~nm})$ in a gap extending to about $100 \mathrm{~nm}$. Nanometer scale gaps are created between the monolayer-protected nanoparticles.

third electrode ${ }^{[13]}$ or via external stimuli such as light or chemicals, as we will describe below.

In the following, we shall first briefly present how individual molecular junctions can be fabricated via mechanically controllable break junctions (BJ). We will also emphasize the importance of performing a statistical analysis to determine the conductance of molecular junctions. The demonstration of stacking effects will be used as an example of the insight that BJs can bring to understanding the formation mechanisms of molecular junctions. In a second experimental approach, we shall see how arrays of metallic nanoparticles represent a useful architecture for building networks of molecular junctions. This platform enables the fabrication of hundreds of molecular junctions in parallel and can be used to test functional molecules. We show here that the electrical properties of the networks can be modulated by up to one order of magnitude, taking advantage of the optical and redox properties of specifically designed compounds.

\section{Contacting Strategies}

The challenge in implementing metalmolecule-metal junctions consists in engineering suitable electrodes. Achieving a two-point or three-point contacting of nanometer-scale molecules is a demanding task. Over the years, various original techniques have been devised and extensively detailed in the literature.[14-16] We will briefly describe here the three approaches illustrated in Fig. 2.

The first technique, mechanicallycontrollable break junctions (BJ), was pioneered by Moreland and Ekin to study superconducting weak links ${ }^{[17]}$ and further developed by van Ruitenbeek et al., for molecular junctions. ${ }^{[18]}$ Here, a free-standing metallic wire with a constriction in its centre is prepared on a flexible substrate and continuously stretched by bending the substrate in a three-point bending mechanism (Fig. 2a). The SEM image shows a free-standing $\mathrm{Au}$ bridge fabricated on a stainless steel substrate via electron-beam lithography. The steel plate was first covered with an insulating polyimide layer, visible immediately below the $\mathrm{Au}$ film. 
The elongation causes the diameter at the constriction to become smaller and smaller until the wire breaks to produce two atomic-scale metallic contacts separated by a few Angstroms only, thereby forming a tunnel junction. ${ }^{[19]}$ This technique offers not only the possibility to tune the gap size with exceptional precision but is also reversible, enabling hundreds of openclose cycles to be performed. Some of the pioneering studies on molecular junctions have been performed using $\mathrm{BJ}$ s but were done in an ultra-high vacuum (UHV) environment, mostly at low temperatures. Working in UHV enables very clean conditions but may induce unwanted alterations of the molecular compounds and makes the assembly of the junctions a challenging task. Adding a liquid cell to a BJ setup, we showed that it is possible to work in a chemically controllable environment, with the solvent behaving essentially as a passive element. ${ }^{[20]}$ We observed that the major effect of the solvent consists in a slight lowering of the effective tunnel barrier height of the junction. This approach has therefore allowed the operation of BJs in liquid, offering the flexibility to adjust the solution during measurements. Chemical reactions for in situ deprotection or change of redox state of the molecule can thus be achieved, providing additional experimental flexibility. We have been exploiting this technique extensively over the past years to determine some of the basic mechanisms at stake in the formation of molecular junctions. ${ }^{[21-29]}$ Some examples are provided in the next section.

Another widely used technique to fabricate nanoscale gaps consists in carrying out an electromigration (EM) process in metallic wires patterned with a constriction as depicted in Fig. 2b. ${ }^{[30]}$ By maintaining a large current density in the wire, a diffusion of the metal atoms can be initiated to thin the wire until it finally breaks open. The process was first applied to prepare molecular junctions by Park et al., ${ }^{[31]}$ and further exploited and refined by others. ${ }^{[13]}$ While the technique has the appearance of simplicity, it requires some care to achieve a proper implementation. A central issue is the local temperature of the junction during the electromigration process, which can reach a few hundred centigrades. ${ }^{[32-34]}$ If molecules are present during the process, such a temperature may cause irreversible damage to the molecular compounds. Elevated temperatures can also lead to the formation of metallic islands in the immediate vicinity of the junction and interfere with any subsequent characterization of the molecular junction. ${ }^{[35]}$ To achieve a smooth gap opening, it is therefore necessary to implement a control of the voltage drop over the junction during the EM process. ${ }^{[32,36]}$ We have shown that adding an electronic feedback in a four-terminal geometry enables an accurate control of EM progression. ${ }^{[30]}$ The technique is fast, on the scale of seconds, and enables the fabrication of nanogaps in $\mathrm{Au}$ electrodes with a yield approaching $90 \%$ or better. An example of a gap produced with this technique is shown in Fig. 2b. The left SEM image shows a typical metallic constriction before the EM process. On the right SEM image, we can observe the formation of a slit, typically a few tens of nanometers wide. The region where a nanoscale gap has opened is indicated by the circle. From this particular image, we can only state that the gap size is below ten nanometers. Recent studies could image the gap formation during the EM process with great accuracy via TEM measurements. ${ }^{[37,38]}$ As compared to $\mathrm{BJ}$, this technique has the advantage to permit an easier implementation of a third (gate) electrode, helping to refine the molecular junction control. It can also be more easily implemented at large scale on Si wafers for instance, enabling the fabrication of tens or hundreds of nanogaps in parallel. However, it is not reversible and requires a very fine control. Once the gap is opened, it cannot easily be closed and re-opened to gather additional statistical information on the junction formation.

Finally, an alternative method to interface molecules and microfabricated electrical contacts consists in using metallic nanoparticles. Starting from electronbeam fabricated microelectrodes separated by a few tens of nanometers, it is possible to close the gap by metallic nanoparticles (NPs) acting as intermediate contacts to link the molecules with the electrodes. [39,40] This approach is depicted in Fig. 2c. The issue here consists in precisely positioning the nanoparticles within the gap. A simple and controllable method to achieve this consists of aligning and contacting the particles by means of dielectrophoresis. ${ }^{[41]}$ This technique has previously been applied to manipulate a variety of micrometer- and nanometer-size objects including metallic colloids ${ }^{[42,43]}$ but also softer objects such as biopolymers. ${ }^{[44]}$ An important aspect consists here in controlling the trapping conditions. Once the NPs bridge the electrodes, a drop in resistance will result which may lead to an increase in current density and a melting of the NPs: the result will be the formation of a nanoscale wire. ${ }^{[45]}$ In order to fabricate molecular junctions, one may start by coating the NPs with the molecules to study. This will lead to the situation depicted in Fig. 2c. It will however be impossible to do so for specific compounds due to solubility issues for instance. In that case, a template structure with alkaneprotected NPs can be prepared. Molecules of interest can later be introduced in the device, replacing the alkane chains via a chemical exchange. ${ }^{[46]}$ We will come back to molecular exchange in the final section where 2D NPs arrays will be discussed.

\section{Assembling a Molecular Junction}

As a model molecular system, we have chosen an oligo-phenylene ethynylene (OPE) compound due to the strong delocalization of its electronic orbitals along the molecular backbone. ${ }^{[47]}$ To enable the bridging of a gap, the molecules bear an anchor group at each end. In this case, a sulfur atom enables a covalent bond formation to the gold as illustrated by the scheme in Fig. 3a. By successively opening and closing nanoscale gaps in a BJ setup, we can bridge the gap via one or a few molecules and form molecular junctions. ${ }^{[24]}$ Representative conductance traces $\mathrm{G}(\mathrm{z})$ obtained during opening cycles are shown in Fig. 3a for the pure solvent (left, grey open squares) and in presence of a $0.25 \mathrm{mM}$ OPE solution (right, black disks). The conductance is plotted as a function of the vertical displacement of the push-rod $\mathrm{z}$ and shown in a logarithmic scale in order to provide a full view of its evolution during an opening cycle.[22] The ratio between the horizontal elongation of the metallic bridge and the vertical push-rod displacement obtained for microfabricated junctions reaches typically $10^{-5}$, giving them an excellent mechanical stability. ${ }^{[20,22]} \mathrm{A}$ vertical displacement of $1 \mu \mathrm{m}$ will thus result in a $10 \mathrm{pm}$ gap widening. During bending and opening of the gap, the conductance $G(z)$ decreases, showing a series of conductance plateaus for $\mathrm{G}$ values above the quantum conductance unit $\mathrm{G}_{0}=2 \cdot \mathrm{e}^{2} / \mathrm{h}$ (arrow in Fig. 3a). A last plateau appears at a value $\mathrm{G} \cong \mathrm{G}_{0}$, which corresponds to the formation of a single-atom gold bridge. If the junction is elongated further, it breaks open, as evidenced by a sudden decrease of conductance down to $\mathrm{G} \sim 10^{-2}$ to $10^{-3}$ $\mathrm{G}_{0}$. This sudden rearrangement of the gold atoms causes a reshaping of the front ends of the two electrodes. The conductance is then governed by electron tunneling which results in a linear dependence of $\log (\mathrm{G})$ versus $\mathrm{z}$ as evidenced by the dashed line. Conductance traces acquired sequentially will be different due to fluctuations in the local geometry of the junction. It is therefore necessary to build conductance histograms of the measured $\log (\mathrm{G})$ values (right side of Fig. 3a) to provide a statistically relevant junction characterization. When OPE molecules terminated with sulfur linkers on both ends are added to the BJ liquid cell, the $G(z)$ dependence changes drastically to exhibit clear conductance plateaus developing at conductance values below $G_{0}$. Such plateaus are the signature for the formation of a molecular junction. About $40 \%$ 
of the traces show a molecular signature in this case. In the $\log (\mathrm{G})$ histogram, the data translate to a broad, but very pronounced peak centered at $\mathrm{G} \cong 1.2 \cdot 10^{-4} \mathrm{G}_{0}{ }^{\cdot}{ }^{[24,27]}$ Representing the data in a $\log (\mathrm{G})$ histogram permits avoiding any data selection. Curves that do not show plateaus will only contribute to a tunneling background similar to that from a pure solvent. ${ }^{[22]}$

As an example of the insight that the technique can bring to understanding molecular junctions, we briefly discuss observations obtained for molecules bearing only one end terminal.[27] With only a single specific anchor, one would a priori not expect the formation of a stable metalmolecule-metal bridge. The data shown in Fig. $3 b$ therefore come as a surprise. The top histogram shows the same data as in Fig. 3a for the dithiolated OPE compound, plotted now horizontally. Quite strikingly, the bottom histogram for monothiolated OPE shows also a very clear peak, indicating the formation of stable molecular junctions. This observation led us to further reflect on the formation mechanisms of molecular junctions. To this point, intermolecular interactions have been disregarded. However, for metallic electrodes with a dense coverage of molecules, it can be expected that more than one molecule will be in the immediate vicinity of the gap, as schematically represented in Fig. 1a. This is further supported by the fact that the junctions are created at room temperature and in a liquid environment, thereby favoring the molecules' mobility. We therefore believe that the formation of a stable metal-molecule-metal system can be driven by $\pi-\pi$ stacking interaction between a pair of adjacent molecules, ${ }^{[48]}$ as depicted in the schematics of Fig. 3b. This interpretation is supported by the shift of the conductance peak to lower values by more than an order of magnitude. Indeed, a reduced conductance value for the junction is expected, because a pair of stacked molecules will be longer than a single dithiolated molecule anchored between gold electrodes, as schematically depicted in Fig. 3b. Recent theoretical and experimental work by other groups ${ }^{[49,50]}$ reinforce this analysis and clearly reveal the importance of the immediate surroundings in transport molecular junctions involving a single or a few molecules. [51-53]

\section{Networks of Molecular Junctions}

If practical molecular devices are to emerge, they will have to be the result of a thorough design and implementation. One aspect is the need for specifically engineered molecules exhibiting e.g. switching properties. ${ }^{[54]}$ Importantly, the functionality of these compounds needs to be

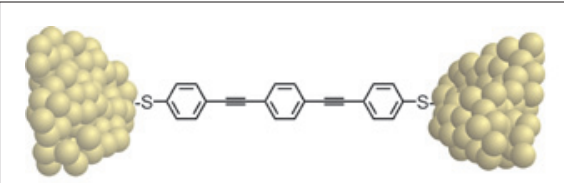

(a)

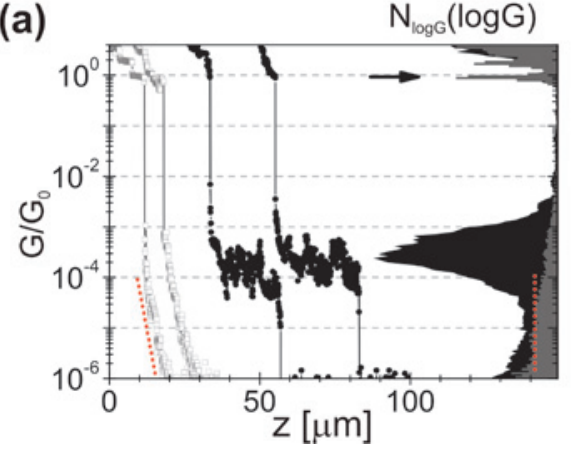

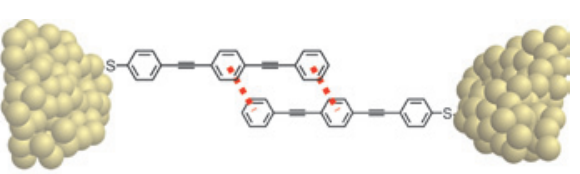

(b)

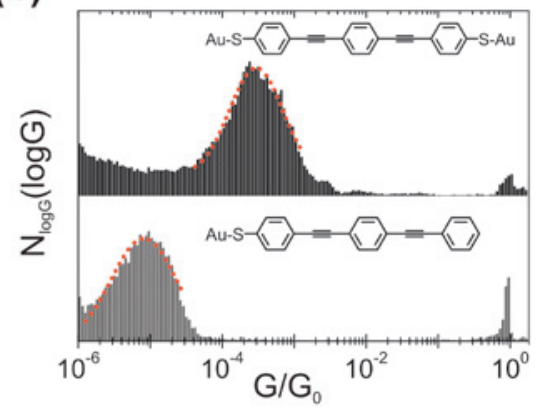

Fig. 3. Measurements of molecular junctions realized with break junctions operated in liquid. (a) Typical conductance traces G(z) measured in pure solvent (open symbols, grey) and in the same solvent with OPE-dithiol molecules (solid symbols, black). The curves are shifted horizontally for clarity. $z$ is the vertical displacement of the push-rod. The solvent was a mixture of tetrahydrofuran/mesitylene (1:4 v/v ratio). On the right axis are shown the histograms of $\log (\mathrm{G})$ values obtained from 100 opening curves each. In the tunneling regime, the solvent contribution results in a constant number of counts in the histogram (dashed lines). In the presence of OPEdithiol molecules, clear plateaus develop in the individual traces and result in a peak signature in the histogram. (b) Comparison of $\log (G)$ histograms for OPE molecules with two linking terminals (black) and with only one thiol linker (grey). Each histogram was built from 100 conductance traces obtained during successive opening cycles. The pronounced Gaussian-like peaks (dashed lines) in the $\log (G)$ histograms represent the signatures of the specific molecule investigated. An average molecular junction conductance is deduced from the peak conductance.

characterized in a junction geometry using techniques such as those described above. Equally important is the need for schemes enabling the implementation of molecular junctions at large scale. ${ }^{[55,56]}$ Here, establishing fabrication protocols taking advantage of molecular self-assembly could be an asset to simplify the process. In this perspective, nanoparticles (NPs) may be ideal candidates to bridge molecules with bulk material ${ }^{[57]}$ and enable the fabrication of stable and upscalable devices. ${ }^{[58]}$ NPs arrays can in particular exhibit a rich diversity of transport properties. ${ }^{[59]}$ Recently, we have shown that 2D NPs arrays form an interesting platform for molecular electronics, while based on a simple self-assembly and microcontact printing approach offering upscaling possibilities. ${ }^{[60-65]}$

Fig. 4a depicts a device based on a NPs array. [60] Two metallic contacts are deposited on top of a 2D array of Au nanoparticles coated with alkane chains. A SEM picture showing two such devices on top of a $\mathrm{Si} /$ $\mathrm{SiO}_{2}$ wafer is presented in Fig. 4b. The contacts have dimensions of $50 \times 50 \mu \mathrm{m}^{2}$ and are $10 \mu \mathrm{m}$ apart. The diameter of the $\mathrm{Au}$ NPs is about $10 \mathrm{~nm}$ while the number of carbon atoms in the thiolated alkane chains ranges between eight and sixteen. The NPs are first self-assembled as an array at an air/water interface and transferred to the substrate via a patterned stamp to prepare stripes typically $20 \mu \mathrm{m}$ wide, as empha- sized by dashed lines. The TEM picture at the bottom of Fig. $4 \mathrm{~b}$ is a detailed view of the two-dimensional array showing the hexagonal packing formed by octanethiolcoated NPs at the surface of the substrate.

At this stage, the nanoparticles are not interconnected: the cohesion of the structure is ensured by the interdigitation of the alkane chains. To form an interconnected network of molecular junctions, we have to insert molecular compounds bridging neighboring NPs. This is done via a molecular exchange process as depicted in Fig. 4c. The substrate bearing the NP devices is immersed in a solution containing, in this case, a dithiolated OPE compound. Upon immersion, the OPE molecules will partially displace the alkane chains ${ }^{[60,61]}$ and interlink neighboring nanoparticles to form a network of molecular junctions. ${ }^{[62]}$ Remarkably, this process is reversible and multiple exchanges can be performed without notably altering the NP array. ${ }^{[60,61]}$ NPs arrays can therefore be seen as a spatially well-defined matrix of nanoscale contacts in which different molecular compounds can be inserted at will. While it is not readily possible to address each junction separately in this geometry, such devices provide a complementary insight to characterize molecular junctions. Techniques such as break junctions enable the successive formation of hundreds of junctions over time to achieve a statistically relevant 


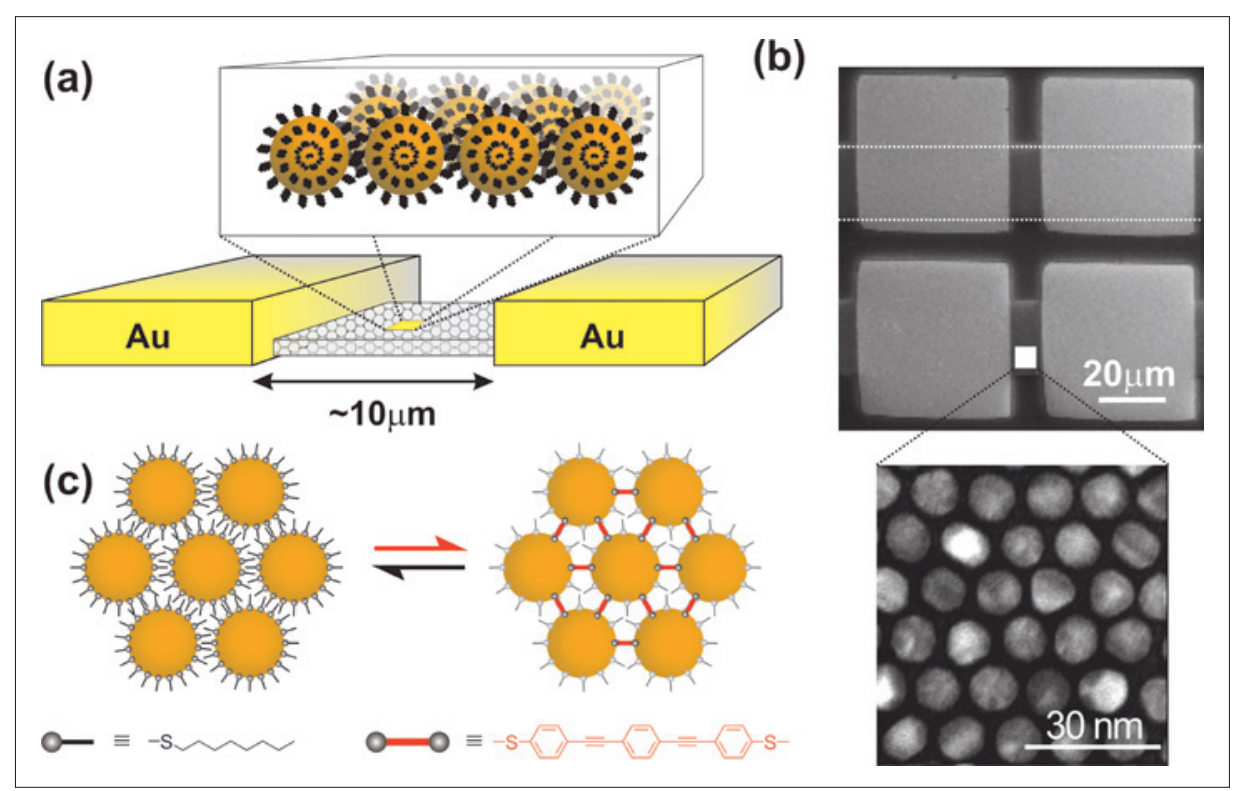

Fig. 4. Devices based on micro-contact printed self-assembled nanoparticle arrays. (a) Schematics of a device. (b) SEM image of two devices (top and bottom) each of which comprises two Au contact pads deposited on a one monolayer-thick nanoparticle array (stripe) between them. The boundaries of the nanoparticle stripes are emphasized on the top device (dashed lines). The high magnification TEM image shows the regular hexagonal packing formed by the nanoparticles. (c) Molecular-exchange process carried out in liquid. Left: self-assembled alkanethiol-capped nanoparticles before exchange; right: after the exchange process. The incoming molecules (red, bottom right) displace part of the alkane chains and interlink neighboring nanoparticles to form a network of molecular junctions.

molecular signature. NP arrays, however, provide a molecular junction signature averaged over hundreds of thousands of junctions. A device like that in Fig. 4b contains typically $\sim 10^{6}$ junctions. The sheet conductance $G_{\square}$ of the array is defined by $G_{\square}$ $=\mathrm{G} \cdot \mathrm{l} / \mathrm{w}$, where $\mathrm{G}$ is the device conductance and $l$ and $w$ are the length and width of the array area comprised between the contact pads. For our two-dimensional structures, the sheet conductance corresponds to the conductance of one junction normalized by a simple geometrical factor. ${ }^{[60]}$ Comparing the conductance of molecular junctions networks to conductance values deduced from BJ experiments for the same molecules, we find that neighboring nanoparticles are bridged by one molecule on average. ${ }^{[60]}$

We have tested the validity of the NP platform for molecules able to respond to light and chemical stimuli. Fig. 5a show a photochromic compound[66] that can reversibly be switched between a conjugated (closed) and a cross-conjugated state (open) using UV and visible light.[63] Importantly, the length change during this isomerization process is negligible, ensuring a minimal stress for the molecular junction upon state change. We inserted the closed form of these compounds in NP arrays using a molecular exchange and performed successive illumination cycles with visible and UV light. A clear alterna- tion of a conductance decrease and increase following visible and UV light irradiation was observed (Fig. 5b). Upon visible light illumination, compounds will switch to the open state. The resulting conjugation interruption will cause a HOMO-LUMO gap increase and we can anticipate, following the simplified picture in Fig. 1b, that the conductance of the array shall decrease, as observed from the data. Alternatively, switch back to the closed state showing a smaller HOMO-LUMO gap. The data show indeed a conductance increase immediately after the illumination started. Several control experiments helped confirming that the conductance modulation is indeed due to the switching of the molecule between the open and closed states. ${ }^{[63]}$ Note that the amplitude of the conductance modulation decreases as the number of cycles increases. This can be explained by irreversible effects such as contact loss or photodecomposition ${ }^{[66]}$ as well as by the fact that we used limited irradiation times in order to prevent photodecomposition. If less than $100 \%$ of the molecules are switched at each illumination cycle, the overall amplitude of the conductance change will decrease with time.

In Fig. 5c, we present a redox-active molecule based on a tetrathiafulvalene (TTF) unit. ${ }^{[65]}$ Redox molecules are particularly attractive candidates for molecuupon UV illumination, the molecules will lar electronics since the current through the junctions can be influenced either by electrochemical gating or by the presence of oxidizing or reducing agents. While several different redox units have been considered so far, TTF compounds have seldom been incorporated in metal-molecule-metal junctions. ${ }^{[25]}$ The stability of the different oxidation states of TTF species makes them attractive for switching purposes and molecular scale logics. Upon exposure to appropriate oxidizing and reducing agents, the TTF unit can be oxidized to the $\mathrm{TTF}^{2+}$ state and reduced back to the neutral state. The TTF compound bearing the thiolated alkane linkers was shown to behave similarly to the basic TTF unit and UV/vis spectroscopy evidenced that the $\mathrm{TTF}^{2+}$ state presents a lower HOMO-LUMO gap than the neutral state. ${ }^{[65]}$ We followed a similar procedure to that described for the photochromic switch and inserted the TTF derivative in C8-capped gold NP arrays and characterized the conductance of the arrays at different experimental stages. In Fig. 5d, we plot the array sheet conductance averaged over 24 devices at different stages of the experiment. The conductance has been normalized to the initial conductance of the array $\mathrm{G}_{\text {neutral }}$ measured immediately after inserting the neutral state TTF compound (first disc data point). The subsequent discs data points are measurements after immersion in excess reducing agent. The squares correspond to measurements after immersion in excess oxidizing agent. After immersion in either agent, the devices were rinsed and dried in order to perform the conductance measurements. Remarkably, we can see that the conductance modulation achieved here by oxidation reached about one order of magnitude. Various control experiments were performed to confirm that the switching behavior can be attributed to the oxidation of the TTF compound in the network of molecular junctions. ${ }^{[65]}$

With these two examples, we demonstrate that the functionality provided by specifically-engineered molecules can be preserved in nanostructured devices where the molecules have been electrically contacted.

\section{Concluding Remarks}

Contacting individual molecules with nanometer-scale electrodes has become a reality in many research laboratories today. By implementing specific techniques, twoand three-terminal devices have been realized and molecular junctions extensively characterized. Moreover, there is currently a substantial effort in the research community to characterize test molecular systems and perform systematic comparisons be- 
(a)

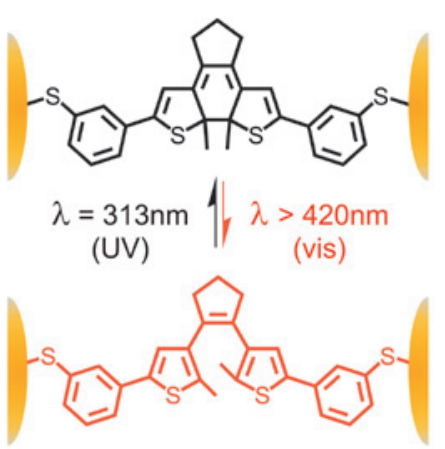

(c)

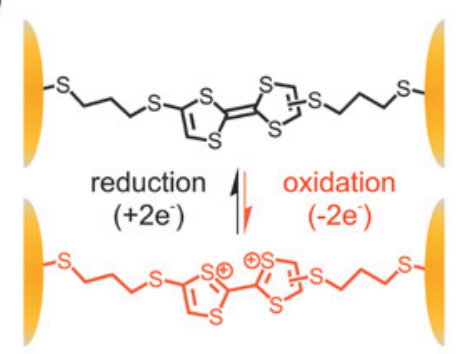

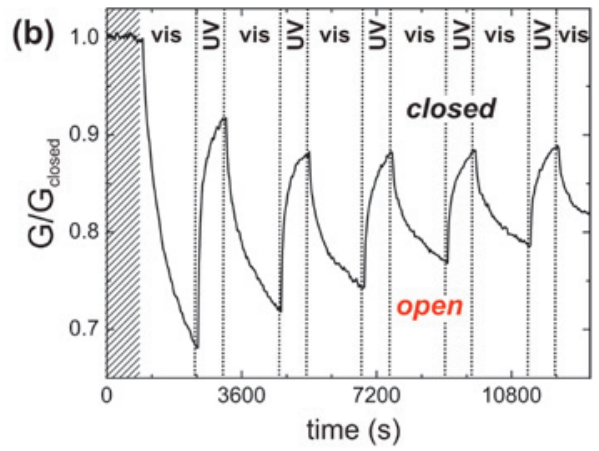

(d)

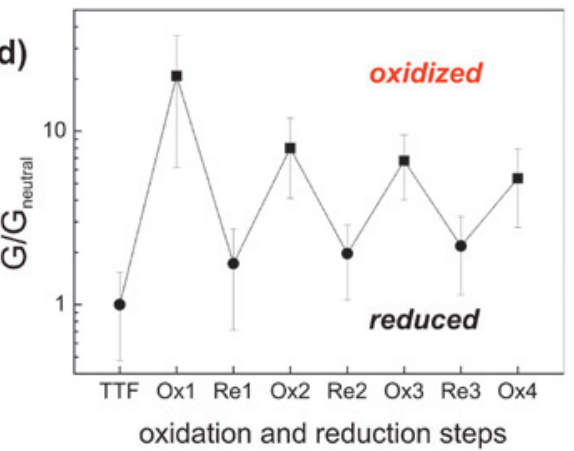

Fig. 5. Example of switchable molecular compounds showing functionality after insertion in NPs array devices. (a) Structure of a photochromic diarylethene molecule in its closed (top) and open (bottom) forms. The switching from closed to open is achieved via illumination with visible light. The molecule can be closed again using UV light. In the closed form, the compound is fully conjugated while in the open form, the conjugation is interrupted. (b) Repeated conductance switching of a NP device containing the photochromic switch. We report the normalized array conductance $G / G_{\text {closed }}$ vs illumination time t. $G_{\text {closed }}$ is the array conductance immediately after insertion of the closed photochromic switch. A clear conductance decrease and increase is observed during successive illumination cycles with visible and UV light. (c) Structure of a redoxactive dithiolated tetrathiafulvalene molecule. In presence of iron chloride or ferrocene, the TTFdT compounds can be oxidized or reduced. (d) Average conductance $G / G_{\text {neutral }}$ for 24 NP devices during four successive cycles of oxidation-reduction. The conductance is plotted as a function of process step, starting from the array immediately after insertion of the TTFdT compound in the neutral state via molecular exchange.

tween different techniques. ${ }^{[67,68]}$ This will be essential to evaluate the feasibility of practical molecular devices. The fact that molecular properties can be maintained in nanoscale devices already demonstrates that a proper combination of physics, chemistry and engineering can lead to the fabrication of functional devices. At this point, it might be appropriate emphasizing that 'functional' for research devices means that, for instance, a switching effect has been observed in a few devices and repeated for a few cycles: achieving hundreds of thousands of reproducible switching events for a large number of devices is a totally different endeavor. The implementation of true molecular-scale logic ${ }^{[69]}$ is therefore probably still far away. Nevertheless, the progress in understanding molecular junctions has been tremendous over the past ten years as the effects investigated no go well beyond simple tunneling. ${ }^{[70]}$ For instance, molecular spintronics $^{[71]}$ or quantum interference effects in molecular junctions ${ }^{[72]}$ are under scrutiny. We can therefore expect this fascinating, interdisciplinary field of molecular electronics to keep researchers intrigued for a number of years.

\section{Acknowledgments}

The work presented here is the result of various fruitful collaborations between physicists and chemists. The author wishes to thank in particular C. Schönenberger for his support throughout the past years. For their essential and valued contributions to the work presented, acknowledgements also go to N. Agraït, J. S. Agustsson, C. R. Arroyo, L. Bernard, G. RubioBollinger, J. Brunner, M. R. Bryce, F. Cheng, S. Decurtins, F. Diederich, B. L. Feringa, F. Giacalone, M. T. González, L. Grüter, S. Grunder, T. T. Heikkilä, M. Ángeles Herranz, R. Huber, A. W. Holleitner, V. Horhoiu, O. Jeannin, R. Jitchati, Y. Kamdzhilov, T. Kudernac, M. Langer, Y. Leroux, J. Liao, S.X. Liu, M. A. Mangold, N. Martin, M. Mayor, S. J van der Molen, Y.-F. Ran, M. Vélez, C. Wang, B. J. van Wees, S. Wu and Z. M. Wu. Fruitful discussions with M. Brandbyge, N. J.
Tao, R. J. Nichols, H. Ottosson, M. A. Ratner, L. Venkataraman and T. Wandlowski are gratefully acknowledged. Technical support by P. Reimann, M. Steinacher and H. Breitenstein is also gratefully acknowledged.

This work was supported by the University of Basel, the Swiss NCCR on Nanoscale Science, the Swiss National Science Foundation, the GEBERT RUF STIFTUNG, the European Science Foundation through the Eurocore program on Self-Organized Nanostructures (SONS) the European Commission (EC) FP7 ITN 'FUNMOLS' project no. 212942 and the EC FP7 'FUNMOL' project no. 213382.

Received: May 3, 2010

[1] S. R. Forrest, Nature 2004, 428, 911; S. R. Forrest, M. E. Thompson, Chem. Rev. 2007, 107, 923.

[2] C. A. Mirkin, M. A. Ratner, Ann. Rev. Phys. Chem. 1992, 34, 719; C. Joachim, J. K. Gimzewski, A. Aviram, Nature 2000, 408, 541; A. Nitzan, Ann. Rev. Phys. Chem. 2001, 52, 681.

[3] M. A. Reed, C. Zhou, C. J. Muller, T. P. Burgin, J. M. Tour, Science 1997, 278, 252; C. Joachim, M. A. Ratner, Proc. Nat. Acad. Sci USA 2005, 102, 8801; M. Galperin, M. A. Ratner, A. Nitzan, J. Phys.: Condens. Matter 2007, 9, 103201.

[4] N. Weibel, S. Grunder, M. Mayor, Org. Biomol. Chem. 2007, 5, 2343.

[5] D. D. Eley, D. I. Spivey, Trans. Farad. Soc. 1962, 58,411 .

[6] B. Mann, H. Kuhn, J. Appl. Phys. 1971, 42, 4398.

[7] A. Avriram, M. A. Ratner, Chem. Phys. Lett. 1974, 29, 277.

[8] F. Zahid, M. Paulsson, S. Datta, 'Electrical Conduction through Molecules', in 'Advanced Semiconductors and Organic Nano-Techniques', Ed. H. Morkoc, Academic Press, 2003.

[9] Y. Selzer, D. L. Allara, Annu. Rev. Phys. Chem. 2006, $57,593$.

[10] J. M. Thijssen, H. S. J. Van der Zant, Phys. Stat. Sol. 2008, 245, 1455.

[11] H. B. Akkerman, B. de Boer, J. Phys.: Condens. Matter 2008, 20, 013001

[12] K. Moth-Poulsen, T. Bjørnholm, Nature Nanotechnol. 2009, 4, 551.

[13] See e.g. the recent review by E. A. Osorio, T. Bjørnholm, J. M. Lehn, M. Ruben, H. S. J. van der Zant, J. Phys.: Condens. Matter 2008, 20, 374121.

[14] H. Haick, D. Cahen, Progress in Surface Science 2008, 83, 217.

[15] N. Prokopuk, K.-A. Son, J. Phys.: Condens. Matter 2008, 20, 374116

[16] R. L. McCreery, A. J. Bergren, Adv. Mat. 2009 , 21,4303 .

[17] J. Moreland, J. W. Ekin, J. Appl. Phys. 1985, 58, 3888.

[18] C. J. Muller, J. M. van Ruitenbeek, L. J. de Jongh, Physica C 1992, 191, 485.

[19] N. Agraït, A. L. Yeyati, J. M. van Ruitenbeek, Phys. Rep. 2003, 377, 81.

[20] L. Grüter, M. T. González, R. Huber, M. Calame, C. Schönenberger, Small 2005, 1, 1067.

[21] L. Grüter, F. Cheng, T. T. Heikkilä, M. T. Gonzalez, F. Diederich, C. Schönenberger, M. Calame, Nanotech. 2005, 16, 2143.

[22] M. T. Gonzalez, S. Wu, R. Huber, S. J. van der Molen, C. Schönenberger, M. Calame, Nano Lett. 2006, 6, 2238.

[23] S. Grunder, R. Huber, V. Horhoiu, M. Gonzalez, C. Schönenberger, M. Calame, M. Mayor, J. Org. Chem. 2007, 72, 8337.

[24] R. Huber, M. Gonzalez, S. Wu, M. Langer, S. Grunder, V. Horhoiu, M. Mayor, M. Bryce, 
C. Wang, R. Jitchati, C. Schönenberger, M. Calame, J. Am. Chem. Soc. 2008, 130, 1080.

[25] F. Giacalone, M. A. Herranz, L. Grueter, M T. Gonzalez, M. Calame, C. Schönenberger, C. R. Arroyo, G. Rubio-Bollinger, M. Velez, N. Agraït, N. Martín, Chem. Comm. 2007, 46, 4854.

[26] M. T. Gonzalez, J. Brunner, R. Huber, S. Wu, C. Schönenberger, M. Calame, New J. Phys. 2008 10, 065018.

[27] S. Wu, R. Huber, M. T. Gonzalez, S. Grunder, M. Mayor, C. Schönenberger, M. Calame, Nature Nanotechnol. 2008, 3, 569.

[28] S. Grunder, R. Huber, S. Wu, C. Schönenberger, M. Calame, M. Mayor, Eur. J. Org. Chem. 2010 5,833 .

[29] S. Grunder, R. Huber, S. Wu, C. Schönenberger, M. Calame, M. Mayor, Chimia 2010, 64, 140.

[30] Z. M. Wu, M. Steinacher, R. Huber, M. Calame, S. J. van der Molen, C. Schönenberger, Appl. Phys. Lett. 2007, 91, 053118.

[31] H. Park, J. Park, A. K. L. Lim, E. H. Anderson, A. P. Alivisatos, P. L. McEuen, Nature 2000 , 407, 57.

[32] D. R. Strachan, D. E. Smith, D. E. Johnston, T.-H. Park, M. J. Therien, D. A. Bonnell, A. T. Johnson, Appl. Phys. Lett. 2005, 86, 43109.

[33] M. L. Trouwborst, S. J. van der Molen, B. J. van Wees, J. Appl. Phys. 2006, 99, 114316.

[34] D. R. Ward, N. J. Halas, D. Natelson, Appl. Phys. Lett. 2008, 93, 213108.

[35] A. A. Houck, J. Labaziewwicz, E. K. Chan, J. A. Folk, I. L. Chuang, Nano Lett. 2005, 5, 1685.

[36] R. Hoffmann, D. Weissenberger, J. Hawecker, D. Stöffler, Appl. Phys. Lett. 2008, 93, 043118.

[37] T. Taychatanapat, K. I. Bolotin, F. Kuemmeth, D. C. Ralph, Nano Lett. 2007, 7, 652 .

[38] H. B. Heersche, G. Lientschnig, K. O’Neill, H. S. J. van der Zant, H. W. Zandbergen, Appl. Phys. Lett. 2007, 91, 072107.

[39] A. S. I. Khondaker, Z. Yao, Appl. Phys. Lett. 2002, 81, 4613.

[40] T. Dadosh, Y. Gordin, R. Krahne, I. Khivrich, D. Mahalu, V. Frydman, J. Sperling, A. Yacoby, I. Bar-Joseph, Nature 2005, 436, 677.

[41] H. Morgan, N. G. Green, 'AC Electrokinetics: Colloids and Nanoparticles', Baldock, England, Research Studies Press Ltd., 2003.
[42] A. Bezryadin, C. Dekker, G. Schmid, Appl. Phys. Lett. 1997, 71, 1273.

[43] K. D. Hermanson, S. O. Lumsdon, J. P. Williams, E. W. Kaler, O. D. Veley, Science 2001, 294, 1082.

[44] F. Dewarrat, M. Calame, C. Schönenberger, Single Mol. 2002, 3, 189.

[45] L. Bernard, M. Calame, S. J. van der Molen, J. Liao, C. Schönenberger, Nanotech. 2007, 18, 235202.

[46] M. J. Hostetler, A. C. Templeton, R. W. Murray, Langmuir 1999, 15, 3782.

[47] E. A. Weiss, M. R. Wasielewski, M.A. Ratner, Top. Curr. Chem. 2005, 257, 103.

[48] See for instance: F. Hoeben, P. Jonkheijm, E. Meijer, A. Schenning, Chem. Rev. 2005, 105, 1491.

[49] L.-L. Lin, J.-C. Leng, X.-N. Song, Z-L. Li, Y Luo, C.-K. Wang, J. Phys. Chem. C 2009, 113, 14474.

[50] S. Martin, I. Grace, M. R. Bryce, C. Wang, R. Jitchati, A. S. Batsanov, S. J. Higgins, C. J. Lambert, R. J. Nichols, J. Am. Chem. Soc., accepted.

[51] J. Taylor, M. Brandbyge, K. Stokbro, Phys. Rev. B 2003, 68, 121101.

[52] A. Szuchmacher Blum, J. G. Kushmerick, S. K. Pollack, J. C. Yang, M. Moore, J. Naciri, R. Shashidhar, B. R. Ratna, J. Phys. Chem. B 2004, 108, 18124.

[53] Y. Selzer, L. Cai, M. A. Cabassi, Y. Yao, J. M. Tour, T. S. Mayer, D. L. Allara, Nano Lett. 2005, 5, 61

[54] S. J. van der Molen, P. Liljeroth, J. Phys.: Condens. Matter 2010, 22, 133001.

[55] J. E. Green, J. W. Choi, A. Boukai, Y. Bunimovich, E. Johnston-Halperin, E. DeIonno, Y. Luo, B. A. Sheriff, K. Xu, Y. S. Shin, H.-R. Tseng, J. F. Stoddart, J. R. Heath, Nature 2007, 445, 414.

[56] P. A. Van Hal, E. C. P. Smits, T. C. T. Geuns, H. B. Akkerman, B. C. De Brito, S. Perissinotto, G. Lanzani, A. J. Kronemeijer, V. Geskin, J. Cornil, P. W. M. Blom, B. De Boer, D. M. De Leeuw, Nature Nanotechnol. 2008, 3, 749.

[57] A. C. Templeton, W. P. Wuelfing, R. W. Murray, Acc. Chem. Res. 2000, 33, 27.

[58] A. Shipway, E. Katz, I. Willner, ChemPhysChem 2000, 1,18 .
[59] A. Zabet-Khosousi, A.-A. Dhirani, Chem. Rev. 2008, 108, 4072.

[60] J. Liao, L. Bernard, M. Langer, C. Schönenberger, M. Calame, Adv. Mat. 2006, 18 , 2444.

[61] L. Bernard, Y. Kamdzhilov, M. Calame, S. van der Molen, J. Liao, C. Schönenberger, J. Phys. Chem. C 2007, 111, 18445 .

[62] J. Liao, M. Mangold, S. Grunder, M. Mayor, C. Schönenberger, M. Calame, New J. Phys. 2008 , 10, 065019.

[63] S. van der Molen, J. Liao, T. Kudernac, J. Agustsson, L. Bernard, M. Calame, B. van Wees, B. Ferringa, C. Schönenberger, Nano Lett. 2009, 9, 76 .

[64] M. Mangold, C. Weiss, M. Calame, A. W Holleitner, Appl. Phys. Lett. 2009, 94, 161104.

[65] J. Liao, J. Agustsson, S. Wu, O. Jeannin, Y.-F Ran, S.-X. Liu, S. Decurtins, Y. Leroux, M. Mayor, C. Schönenberger, M. Calame, Nano Lett. 2010, 10, 759.

[66] M. Irie, Chem. Rev. 2000, 100, 1685.

[67] A. Salomon D. Cahen, S. Lindsay, J. Tomfohr, V. B. Engelkes, C. D. Frisbie, Adv. Mat. 2003, 15,1881 .

[68] S. M. Lindsay, M. A. Ratner, Adv. Mat. 2007, $19,23$.

[69] M. H. van der Veen, M. T. Rispens, H. T Jonkman, J. C. Hummelen, Adv. Func. Mat 2004, 14, 215.

[70] M. Galperin, M. A. Ratner, A. Nitzan, A. Troisi, Science 2008, 319, 1056.

[71] L. Bogani, W. Wernsdorfer, Nature Materials 2008, 7,179 .

[72] G.C. Solomon, D. Q. Andrews, R. H. Goldsmith, T. Hansen, M. R. Wasielewski, R. P. Van Duyne, M. A. Ratner, J. Am. Chem. Soc. 2008, 130, 17301 . 